Renée A. Shellhaas, MD, MS

Brian J. Thelen, PhD Jayapalli R. Bapuraj, MD Joseph W. Burns, PhD

Aaron W. Swenson, MD

Mary K. Christensen, BA, RRT

Stephanie A. Wiggins, MS John D.E. Barks, MD

Correspondence to Dr. Shellhaas: shellhaa@med.umich.edu

\section{Limited short-term prognostic utility of cerebral NIRS during neonatal therapeutic hypothermia}

ABSTRACT

Objective: We evaluated the utility of amplitude-integrated EEG (aEEG) and regional oxygen saturation $\left(\mathrm{rSO}_{2}\right)$ measured using near-infrared spectroscopy (NIRS) for short-term outcome prediction in neonates with hypoxic ischemic encephalopathy (HIE) treated with therapeutic hypothermia.

Methods: Neonates with HIE were monitored with dual-channel aEEG, bilateral cerebral NIRS, and systemic NIRS throughout cooling and rewarming. The short-term outcome measure was a composite of neurologic examination and brain MRI scores at 7 to 10 days. Multiple regression models were developed to assess NIRS and aEEG recorded during the 6 hours before rewarming and the 6 -hour rewarming period as predictors of short-term outcome.

Results: Twenty-one infants, mean gestational age $38.8 \pm 1.6$ weeks, median 10-minute Apgar score 4 (range 0-8), and mean initial pH $6.92 \pm 0.19$, were enrolled. Before rewarming, the most parsimonious model included 4 parameters (adjusted $R^{2}=0.59 ; p=0.006$ ): lower values of systemic $\mathrm{rSO}_{2}$ variability $(p=0.004)$, aEEG bandwidth variability $(p=0.019)$, and mean aEEG upper margin $(p=0.006)$, combined with higher mean aEEG bandwidth (worse discontinuity; $p=$ $0.013)$, predicted worse short-term outcome. During rewarming, lower systemic $\mathrm{rSO}_{2}$ variability $(p=0.007)$ and depressed aEEG lower margin $(p=0.034)$ were associated with worse outcome (model-adjusted $R^{2}=0.49 ; p=0.005$ ). Cerebral NIRS data did not contribute to either model.

Conclusions: During day 3 of cooling and during rewarming, loss of physiologic variability (by systemic NIRS) and invariant, discontinuous aEEG patterns predict poor short-term outcome in neonates with HIE. These parameters, but not cerebral NIRS, may be useful to identify infants suitable for studies of adjuvant neuroprotective therapies or modification of the duration of cooling and/or rewarming. Neurology ${ }^{\circledR}$ 2013;81:249-255

\section{GLOSSARY}

aEEG = amplitude-integrated EEG; HIE = hypoxic ischemic encephalopathy; $\mathbf{N I C U}=$ neonatal intensive care unit; $\mathbf{N I R S}=$ near-infrared spectroscopy; $\mathbf{r S O}_{2}=$ regional oxygen saturation; TOI = tissue oxygenation index.

Therapeutic hypothermia is now standard for neonates with hypoxic ischemic encephalopathy (HIE), but adverse outcomes remain common. ${ }^{1-3}$ High-risk infants identified before or during rewarming could be suitable candidates for studies of adjuvant neuroprotective therapies or modified duration of cooling and/or rewarming.

Cerebral perfusion and oxygenation are key biomarkers of brain metabolism and may be disrupted in neonates with HIE. ${ }^{4}$ Near-infrared spectroscopy (NIRS) provides reproducible, quantitative measures of cerebral blood volume (reflecting perfusion) and regional oxygen saturation $\left(\mathrm{rSO}_{2}\right)$, which could represent cerebral metabolism. NIRS measures can also reflect renal, ${ }^{5}$ splanchnic, ${ }^{6}$ and peripheral tissue ${ }^{7}$ perfusion in critically ill infants.

Amplitude-integrated EEG (aEEG) has prognostic value in the first hours after neonatal asphyxia, ${ }^{8-10}$ but hypothermia alters its predictive utility. ${ }^{11,12}$ Furthermore, prediction of outcome utilizing the neurologic examination is modified for neonates treated with therapeutic hypothermia. ${ }^{13,14}$

From the Departments of Pediatrics and Communicable Diseases (R.A.S., M.K.C., S.A.W., J.D.E.B.) and Radiology (J.R.B.), University of Michigan, Ann Arbor; Michigan Tech Research Institute (B.J.T., J.W.B.), Ann Arbor and Children's Hospital and Clinics of MinnesotaMinneapolis (A.W.S.), Minnesota Neonatal Physicians, Minneapolis.

Go to Neurology.org for full disclosures. Funding information and disclosures deemed relevant by the authors, if any, are provided at the end of the article. 
We hypothesized that a combination of monitoring modalities recorded late in the cooling and rewarming protocol would provide more precise predictive value than any single neuromonitoring strategy. Previous work defined several markers of adverse outcomes, ${ }^{11,15}$ but those data were applied primarily to inform discussions of goals of care (i.e., transition to comfort care for infants with little likelihood of survival without severe disability) rather than to identify opportunities for new therapeutic strategies. Identification of accurate markers of good (or unfavorable) prognosis immediately before or during rewarming is a critical prerequisite for the design of interventions that prolong cooling and/or rewarming or that assess the effect of adjuvant neuroprotective therapies for HIE.

METHODS Standard protocol approvals, registrations, and patient consents. This research was approved by the Institutional Review Board of the University of Michigan. Term or near-term neonates (estimated gestational age $\geq 36$ weeks) admitted to our neonatal intensive care unit (NICU) whose parents consented to therapeutic whole-body hypothermia for treatment of HIE, from January 2009 through February 2011, were candidates for participation. Every eligible family agreed to partake in this study and provided written informed consent.

Infants. The cooling procedure, including inclusion criteria, followed a published protocol ${ }^{3}$ : using a cooling blanket, the infants were cooled to $33.5^{\circ} \mathrm{C} \pm 0.5^{\circ} \mathrm{C}$ for 72 hours. Rewarming via the blanket occurred at a rate of $0.5^{\circ} \mathrm{C}$ per hour until normothermia was achieved. This process typically took 6 hours.

Demographic and clinical information, including standardized neurologic examinations, ${ }^{16}$ gestational age, intrapartum events, and medications, was collected. Body temperature, pulse oximetry, and blood pressure were recorded according to the NICU protocol. These values were downloaded and integrated into the NIRS datasets.

Using neonatal NIRS sensors over bilateral parietal regions, as well as one sensor over the thigh as a systemic control, NIRS monitoring was initiated as soon as possible after consent was obtained (Invos 5100C; Somanetics Corp., Troy, MI). Subjects were monitored with NIRS for the duration of therapeutic hypothermia, 6 hours of rewarming, and an additional 12 hours of normothermia. $\mathrm{rSO}_{2}$ was recorded every 5 seconds throughout this period and these data were extracted using proprietary software (Somanetics Corp.). Because the clinical implications of NIRS data are unknown in this population, the results were analyzed off-line and were not available to the treating clinicians.

At the same time as NIRS sensors were applied, dual-channel aEEG monitoring was initiated (BRM-2; BrainZ Instruments, Auckland, NZ). The aEEG monitoring continued throughout the study period (including hypothermia, rewarming, and normothermia phases). The aEEG was reviewed regularly by the investigators and by the clinical treatment team according to standard clinical practice in our NICU. Off-line objective analyses using Analyze Research (version 1.4; BrainZ Instruments) included extraction of the mean voltage amplitude and upper and lower margins of the voltage band (and the difference between these values, reported as the width of the aEEG band). These values, rather than scores of aEEG background by pattern recognition, were selected to allow quantitative, objective, and reproducible statistical analyses of continuous variables instead of ordinal or dichotomous regression models, thereby improving overall statistical power.

All infants were monitored with conventional video-EEG for the final 24 hours of the neuromonitoring study, including approximately 8 hours before rewarming, the 6-hour rewarming phase, and 8 to 12 hours after rewarming. When the treating clinicians suspected seizures, either clinically or by aEEG, conventional video-EEG monitoring was initiated sooner and continued until at least 8 hours after rewarming (in this instance, aEEG was continued for research purposes).

Standardized neurologic examinations were performed on the day of study enrollment and immediately after rewarming (day 4 of life). Examination findings were analyzed by computing Thompson scores. ${ }^{16}$ Low Thompson scores $(<10)$ have been associated with favorable prognosis among infants with HIE, before the therapeutic hypothermia era, and similar neurologic examination findings have been reported to correlate with 18- to 22-month neurodevelopmental outcome after therapeutic hypothermia. ${ }^{14}$

Most subjects underwent a clinically indicated brain MRI scan at 7 to 10 days of life. These studies were systematically evaluated by an experienced neuroradiologist (J.R.B.) and scored according to published criteria, ${ }^{17,18}$ which have been reported to be predictive of neurodevelopmental outcome at 12 months. ${ }^{18}$ Lower scores were assigned for normal MRI studies, with higher scores indicating more extensive injury, as follows: score $0=$ normal; score 1 = abnormal basal ganglia signal; score 2 = abnormal signal in the cortex; score $3=$ abnormal signal in the basal ganglia and the watershed cortex; and score $4=$ abnormal signal in the basal ganglia and in the cortex, extending beyond the watershed area.

Because both examination and imaging data are relevant markers of cerebral injury, we used a composite score as the primary short-term outcome measure. The composite short-term outcome score was determined via a normalized principal component analysis $\left.{ }^{19}: 1\right)$ normalizing both sets of scores by their individual SDs, and 2) adding the normalized scores to derive the composite score. Because this was a linear combination of normalized scores, values could be either positive or negative. This provided a range of potential outcome scores, rather than dichotomizing the infants' neurologic status into "favorable" or "unfavorable" risk categories, thereby enhancing the statistical power of our study.

Pearson correlation coefficients were calculated to assess the correlation between individual physiologic variables and shortterm outcome scores. Multiple regression models were developed to assess NIRS and aEEG data recorded during the 6 hours before rewarming, as well as the rewarming period, as predictors of shortterm composite outcome scores. Regression analyses were computed with trimmed data to reduce artifact (i.e., the top $2.5 \%$ and bottom $2.5 \%$ of the raw $\mathrm{rSO}_{2}$ values for each subject were omitted). The final models were selected based on adjusted $R^{2}$ and parsimony.

RESULTS Twenty-one patients (14 males) were enrolled in this study. Three infants with favorable neurologic status were transferred back to their referring hospitals for convalescence without obtaining MRI and were therefore excluded from the correlation 
and regression analyses because composite outcome scores were not available. Demographic and relevant clinical data for the remaining 18 infants are presented in table 1. Two infants died after redirection of care when their neurologic status remained markedly abnormal after rewarming was complete. Including these 2 , there were 8 subjects with day 4 Thompson scores $\geq 10(38 \%)$.

Thompson scores correlated with MRI severity scores, but the relationship was moderate $\left(r^{2}=0.49\right.$, $p=0.001)$, suggesting that the 2 assessments are complementary rather than duplicative. Among the 11 infants with normal brain MRIs (MRI score $=0$ ), only 2 had completely normal examinations (day 4 Thompson score $=0$ ), whereas 3 had markedly abnormal Thompson scores $(\geq 10)$. The 3 infants with markedly abnormal MRIs (MRI score $=4$ ) all had day 4 Thompson scores $\geq 16$. This imperfect relationship between encephalopathy scores and MRI scores has been described previously, particularly among neonates with moderate HIE. ${ }^{20}$

Results of Pearson correlation analyses are presented in table 2. In univariate analysis, absolute values of cerebral and systemic $\mathrm{rSO}_{2}$ before and during rewarming did not correlate with short-term outcome scores $(p>0.05)$. Cerebral $\mathrm{rSO}_{2}$ variability was also independent from short-term outcome $(p>0.05)$. However, both before rewarming and during rewarming, the

\begin{tabular}{|c|c|c|}
\hline Table 1 & \multicolumn{2}{|c|}{$\begin{array}{l}\text { Demographic and clinical profile of the } 18 \text { subjects with both } \\
\text { examination and brain MRI scores available for analysis }\end{array}$} \\
\hline \multicolumn{2}{|c|}{ Gestational age } & $39.9 \pm 1.6 w k$ \\
\hline \multicolumn{2}{|l|}{ Sex } & $6 \mathrm{~F}, 12 \mathrm{M}$ \\
\hline \multicolumn{2}{|c|}{ Birth weight } & $3,501 \pm 445 \mathrm{~g}$ \\
\hline \multicolumn{2}{|c|}{ Head circumference } & $35.6 \pm 2.0 \mathrm{~cm}$ \\
\hline \multicolumn{2}{|c|}{ Median 5-min Apgar score (range) } & $3(0-7)$ \\
\hline \multicolumn{2}{|c|}{ Median 10-min Apgar score (range) } & $4(0-8)$ \\
\hline \multicolumn{2}{|l|}{ Initial pH } & $6.94 \pm 0.18$ \\
\hline \multicolumn{2}{|c|}{ Initial base deficit } & $19.2 \pm 6.0$ \\
\hline \multicolumn{2}{|c|}{ Seizures on conventional EEG } & $\mathrm{n}=4^{\mathrm{b}}$ \\
\hline \multicolumn{2}{|c|}{ Seizures on amplitude-integrated EEG only } & $\mathrm{n}=3^{\mathrm{b}}$ \\
\hline \multicolumn{2}{|c|}{ Treatment with $\geq 1$ dose of phenobarbital } & $\mathrm{n}=10$ \\
\hline \multicolumn{2}{|c|}{ Thompson score $<10$} & Immediately post-rewarming $\mathrm{n}=10$ \\
\hline \multirow{5}{*}{\multicolumn{2}{|c|}{ MRI scores }} & $0: n=11$ \\
\hline & & $1: n=2$ \\
\hline & & $2: n=2$ \\
\hline & & $3: n=0$ \\
\hline & & $4: n=3$ \\
\hline \multicolumn{2}{|c|}{ Median composite short-term outcome score ${ }^{\mathrm{C}}$ (range) } & $-0.45(-1.45$ to +2.75$)$ \\
\hline
\end{tabular}

variability of systemic $\mathrm{rSO}_{2}$ was the single best predictor of short-term outcome scores $(p=0.04$ for both time periods). None of the aEEG variables were significantly correlated with outcome in univariate correlation analyses. However, several of these parameters had correlations that approached statistical significance and/or were considered potentially clinically useful variables, so they were incorporated in the multivariate model-building process.

For data recorded during the 6 hours before rewarming, the most parsimonious multivariate regression model, which predicted outcome with the best statistical significance, included 4 parameters (model-adjusted $R^{2}=0.59 ; p=0.006$ ): lower values of systemic $\mathrm{rSO}_{2}$ variability, aEEG bandwidth variability, and mean upper margin of the aEEG, combined with higher mean aEEG bandwidth (worse discontinuity), predicted worse short-term outcome (table 3). Cerebral NIRS data did not contribute to the model.

The distributions of systemic $\mathrm{rSO}_{2}$ variability and aEEG bandwidth variability during the 6 hours before rewarming are presented in the figure, $A$. The systemic $\mathrm{rSO}_{2} \mathrm{SD}$ did not vary across mean systemic $\mathrm{rSO}_{2}$ values (adjusted $R^{2}=-0.04, p=0.59$; figure, $\mathrm{B}$ ), but aEEG bandwidth SD tended to increase as the absolute value of the bandwidth increased (adjusted $R^{2}=0.73, p<0.0001$; figure, C).

For data recorded during rewarming, the best model included 2 parameters (model-adjusted $R^{2}=$ $0.49 ; p=0.005$ ): lower systemic $\mathrm{rSO}_{2}$ variability and lower aEEG lower margin predicted worse short-term outcome (table 4). Again, cerebral $\mathrm{rSO}_{2}$ data did not contribute to the model.

DISCUSSION This study demonstrates the feasibility of combining aEEG and NIRS, along with conventional EEG monitoring, during therapeutic hypothermia and rewarming in critically ill neonates. In multivariate models, objective aEEG measurements correlated with subsequent short-term neurologic examination and brain MRI scores; these trends were similar to results of previous aEEG studies that used pattern-recognition classification. ${ }^{12}$ However, unexpectedly, cerebral $\mathrm{rSO}_{2}$ data did not contribute to the predictive models. Conversely, we show here that reduced systemic $\mathrm{rSO}_{2}$ variability, which provides a biomarker of the severity of systemic illness, may provide useful prognostic information for neonates with HIE.

Use of NIRS to measure perfusion of renal, splanchnic, and limb tissue has been reported in pediatric and neonatal critical care settings. ${ }^{5-7,21}$ In these scenarios, NIRS may provide real-time data that predict risk of severe medical complications, such as renal failure in newborns with congenital heart disease ${ }^{5}$ and necrotizing enterocolitis in preterm infants. ${ }^{6}$ 


\begin{tabular}{|c|c|c|c|c|}
\hline \multirow{3}{*}{$\begin{array}{l}\text { Table } 2 \\
\text { Variable }\end{array}$} & \multirow{2}{*}{\multicolumn{2}{|c|}{$\begin{array}{l}\text { tions between NIRS and aEEG } \\
\text { Six hours before rewarming }\end{array}$}} & \multirow{2}{*}{\multicolumn{2}{|c|}{ and composite physical examin }} \\
\hline & & & & \\
\hline & Pearson correlation coefficient & p & Pearson correlation coefficient & $p$ \\
\hline Mean cerebral $\mathrm{rSO}_{2}$ & 0.14 & 0.61 & 0.14 & 0.63 \\
\hline Cerebral $\mathrm{rSO}_{2} \mathrm{SD}$ & -0.03 & 0.93 & -0.09 & 0.77 \\
\hline Mean systemic $\mathrm{rSO}_{2}$ & 0.092 & 0.74 & 0.09 & 0.75 \\
\hline Systemic $\mathrm{rSO}_{2} \mathrm{SD}$ & -0.51 & 0.04 & -0.61 & 0.04 \\
\hline Mean aEEG upper margin & -0.38 & 0.15 & -0.39 & 0.13 \\
\hline Mean aEEG lower margin & -0.45 & 0.08 & -0.46 & 0.07 \\
\hline Mean aEEG bandwidth & -0.28 & 0.29 & -0.28 & 0.28 \\
\hline Mean aEEG bandwidth SD & -0.22 & 0.40 & -0.23 & 0.40 \\
\hline
\end{tabular}

Abbreviations: $\mathrm{aEEG}=$ amplitude-integrated EEG; $\mathrm{NIRS}=$ near-infrared spectroscopy; $\mathrm{rSO}_{2}=$ regional oxygen saturation .

However, to our knowledge, systemic NIRS has not been evaluated as a predictor of neurologic outcomes among infants with HIE or other critical illness. Systemic illness is known to increase risk of adverse neurodevelopment among critically ill preterm infants, ${ }^{22}$ and acute kidney injury is associated with longer length of stay and need for assisted ventilation among cooled infants with HIE. ${ }^{23}$ Infants with HIE who are treated with therapeutic hypothermia often have extensive multiorgan dysfunction. ${ }^{24}$ We speculate that this is reflected in reduced systemic $\mathrm{rSO}_{2}$ variability and show that this is predictive of short-term outcome, independent from neuromonitoring parameters. Decreased physiologic variability is reported to be a marker of critical illness. For example, abnormal heart rate variability has been associated with increased morbidity in preterm infants ${ }^{25}$ and with mortality among critically ill children. ${ }^{26}$

Our finding that cerebral NIRS data did not contribute to the multivariate models for prediction of short-term outcome is in contrast to a study published before the era of therapeutic hypothermia. ${ }^{4}$ That study demonstrated increasing $\mathrm{rSO}_{2}$ values during the first 48 hours of life among 9 infants who died and one with adverse neurodevelopmental outcome,

\begin{tabular}{|lll|}
\hline Table 3 & $\begin{array}{l}\text { For the } 6 \text { hours before rewarming, the best predictive model included } \\
\text { these parameters }{ }^{a}\end{array}$ \\
Predictor & Estimate (standard error; $95 \% \mathrm{Cl})$ & p \\
Systemic $\mathrm{rSO}_{2}$ SD & $-0.21(0.06 ;-0.34$ to -0.08$)$ & 0.004 \\
Mean aEEG upper margin & $-1.2(0.35 ;-1.97$ to -0.43$)$ & 0.006 \\
Mean aEEG bandwidth & $2.44(0.83 ; 0.61$ to 4.27$)$ & 0.013 \\
SD of aEEG bandwidth & $-3.8(1.4 ;-6.88$ to -0.72$)$ & 0.019
\end{tabular}

Abbreviations: $\mathrm{aEEG}=$ amplitude-integrated $\mathrm{EEG} ; \mathrm{Cl}=$ confidence interval; $\mathrm{rSO}_{2}=$ regional oxygen saturation.

${ }^{a}$ Model statistics: residual standard error $=0.769$ on $11 d f$; multiple $R^{2}=0.70$; adjusted $R^{2}=0.59 ; p=0.006$ reflecting decreasing oxygen extraction, compared with normal oxygen extraction (stable $\mathrm{rSO}_{2}$ levels) among 8 infants who survived with normal outcomes. Our analysis focused on a later time point, namely the 6 hours before rewarming and the rewarming period (approximately 74-90 hours of life). It is possible that $\mathrm{rSO}_{2}$ values, which others showed to diverge in the first 2 days of life, ${ }^{4}$ could converge again over time. There is also likely an effect of therapeutic hypothermia on NIRS values. For example, cooling could slow brain metabolism such that less oxygen is extracted even among infants with relatively uninjured brains. In this scenario, cerebral $\mathrm{rSO}_{2}$ might not distinguish those with appropriately slowed cerebral metabolism from those with severe brain injury and resultant inability to extract oxygen (luxury perfusion). In this instance, NIRS measures might not diverge until long after rewarming, when cell fate and brain injury become manifest.

A discontinuous, suppressed aEEG pattern and a lack of aEEG variability, predictors of poor shortterm outcome among our subjects, are analogous to the low-voltage/undifferentiated patterns and lack of sleep-wake cycling described by others for infants with HIE who experienced poor outcomes after therapeutic hypothermia. ${ }^{11,12}$ Our data provide quantitative, objective confirmation of these findings, rather than relying on potentially subjective interpretation of the aEEG through pattern-recognition ${ }^{27}$ or voltage criteria ${ }^{8}$ methods, both of which can have limited interrater reliability. ${ }^{28}$

A study of concurrent NIRS and aEEG monitoring among 12 infants with HIE who underwent therapeutic hypothermia demonstrated higher cerebral tissue oxygenation index (TOI) (analogous to $\mathrm{rSO}_{2}$ ) among the 4 who either died or had cerebral palsy by age 1 year, compared with the 8 who had favorable outcomes. ${ }^{15}$ For that study, NIRS was measured from a single sensor over the frontal region and the mean 
A

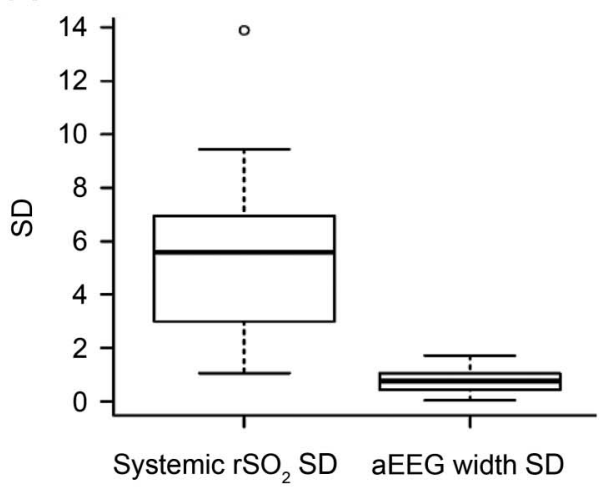

B

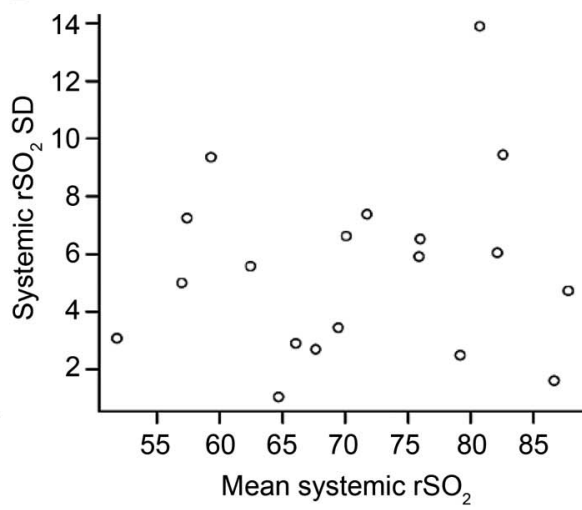

C

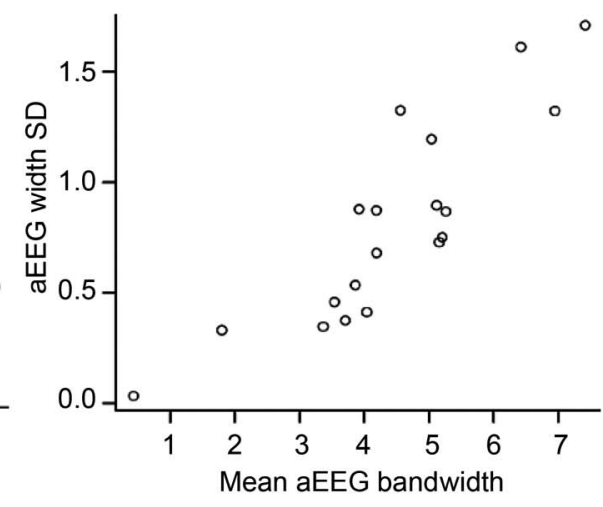

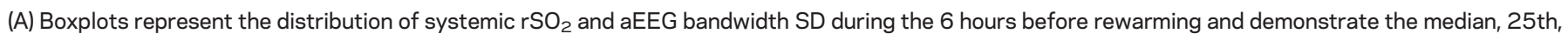

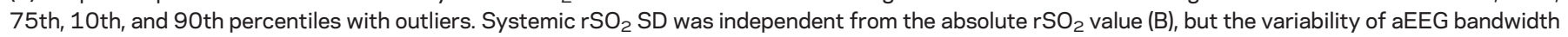
increased as bandwidth increased (C). aEEG = amplitude-integrated $E E G ; r_{2} \mathrm{O}_{2}=$ regional oxygen saturation.

TOI was analyzed at 6,12 , and 24 hours of life; systemic NIRS was not recorded. The authors postulated that higher cerebral TOI could indicate luxury cerebral perfusion among those with poor outcomes. In our study, the absolute values of cerebral $\mathrm{rSO}_{2}$ did not predict short-term outcome in univariate analyses, and only systemic $\mathrm{rSO}_{2}$ variability was included in the final multivariate models. There are several methodologic differences between the 2 studies, which make direct comparisons somewhat difficult: we used bilateral NIRS sensors over the parietal regions (over the cerebrovascular watershed area) whereas they measured NIRS values over the frontal region; we focused the present analysis on the third day of cooling and the rewarming period rather than the first day of life; and our outcome variables are the composite brain MRI scores and Thompson scores rather than dichotomous outcomes, either favorable or adverse (death, cerebral palsy, or abnormal global quotient on the Griffiths scale).

Typically, published cerebral NIRS studies have not reported which type of NIRS sensor was used, although the brand of NIRS monitor is invariably included in the methods section. ${ }^{4,7,15}$ We monitored our subjects with recently developed US Food and Drug Administration-approved neonatal systemic and

\begin{tabular}{|c|c|c|c|}
\hline Table 4 & \multicolumn{3}{|c|}{$\begin{array}{l}\text { For data recorded during rewarming, the best predictive model } \\
\text { included these parameters }{ }^{a}\end{array}$} \\
\hline Predictor & & Estimate (standard error; $95 \% \mathrm{Cl}$ ) & p \\
\hline \multicolumn{2}{|c|}{ Systemic $\mathrm{rSO}_{2} \mathrm{SD}$} & $-0.19(0.06 ;-0.32$ to -0.06$)$ & 0.007 \\
\hline \multicolumn{2}{|c|}{ Mean aEEG lower margin } & $-0.41(0.17 ;-0.78$ to -0.04$)$ & 0.034 \\
\hline
\end{tabular}

Abbreviations: aEEG = amplitude-integrated EEG; $\mathrm{Cl}=$ confidence interval; $\mathrm{rSO}_{2}=$ regional oxygen saturation.

a Model statistics: residual standard error $=0.86$ on $13 d f$; multiple $R^{2}=0.56$; adjusted $R^{2}=0.49 ; p=0.005$ cerebral NIRS sensors (Somanetics Corp.) because these are most likely to be used both in American clinical practice and in future clinical trials. However, other investigators have used older-model pediatric sensors; there is emerging evidence that the recorded $\mathrm{rSO}_{2}$ is not uniform across sensor types (Petra Lemmers and Mona Toet, personal communication, December 2012), and this likely contributes to variation in reported results. Future published NIRS data should specify which sensors were used.

Relatively few of our study subjects (19\%, or 4 of 21) had electrographic seizures confirmed with conventional EEG. This incidence of seizures is lower than that reported by other groups $(30 \%-65 \%) .{ }^{29,30}$ Unless clinically indicated, we monitored subjects with only aEEG until 8 hours before rewarming and then added full conventional EEG monitoring until after rewarming was completed. Given the limited sensitivity of aEEG for seizure detection, it is possible that some seizures were missed. ${ }^{31,32}$ However, status epilepticus is more likely to be detected than isolated seizures on aEEG, and we used dualchannel aEEG with available "raw" EEG, which should improve sensitivity. ${ }^{31}$

Even with conventional EEG monitoring, none of our patients had seizures during the rewarming period. Although animal data raised concern for seizures arising de novo during rewarming, ${ }^{33}$ this phenomenon has not been borne out in the published human studies. In one published $\operatorname{cohort}^{30}(n=26)$, there were no incident seizures during the rewarming period, although some subjects with seizures during hypothermia had persistent seizures as they were rewarmed. Another study ${ }^{29}$ reported that only 1 of 41 infants had seizure emergence during rewarming, whereas 13 of 14 infants with seizures developed their seizures within 18 hours of the beginning of EEG monitoring. 
Our study has some limitations. We describe a very dense dataset acquired from a relatively small cohort of patients, albeit similar in number to other published studies of neuromonitoring for infants with HIE. 4,15,30 A larger sample size could decrease the overall variability and increase the accuracy of the results. Despite this, our sample was sufficient for the analyses presented here. We used a target of $>10 d f$ for our multivariate models. ${ }^{34}$ The models we present in this report have 11 and $13 d f$, thus we are confident that the analysis is appropriate despite the relatively small sample size. Many of our subjects were treated with phenobarbital, lorazepam, or morphine. Each of these might affect cerebral and systemic $\mathrm{rSO}_{2}$. However, similar to the previously published series, ${ }^{4,15}$ our study was not powered to assess such effects.

Our focus for the present analyses has been the period surrounding rewarming, but it is possible that data recorded earlier in the clinical course could have important physiologic and prognostic implications. Further study of the NIRS data recorded in the first 24 to 48 hours of life may reveal additional patterns of interest. Also, we used short-term outcome markers, brain MRI and post-rewarming neurologic examination scores, both of which have been shown to correlate to 12 - to 22-month neurodevelopmental outcome, ${ }^{14,18}$ but we recognize that longer-term neurodevelopmental follow-up will be important for future studies.

Multimodality monitoring for infants treated with therapeutic hypothermia for HIE is feasible and can offer rich data by which to estimate prognosis and guide additional treatment. Our data were recorded in real time and analyzed off-line; additional work is required before these findings could be applied directly at the bedside. In our dense dataset, aEEG provided the best neuromonitoring parameters to predict short-term outcome, and inclusion of systemic NIRS data improved the statistical models. However, the value of cerebral NIRS monitoring remains uncertain in this patient population.

\section{AUTHOR CONTRIBUTIONS}

Dr. Shellhaas designed the study, interpreted the data, and drafted the manuscript. Dr. Thelen analyzed the data and assisted in writing and revising the manuscript. Dr. Bapuraj interpreted the MRIs and assisted in writing and revising the manuscript. Dr. Burns analyzed the data and assisted in writing and revising the manuscript. Dr. Swenson, Ms. Wiggins, and Ms. Christensen assisted in study design and revising the manuscript. Dr. Barks assisted in the study design, data interpretation, and revising the manuscript.

\section{ACKNOWLEDGMENT}

The authors are grateful to the patients and families who participated in this research, as well as the NICU nurses and EEG technologists who facilitated the monitoring protocols.

\section{STUDY FUNDING}

This research was supported by the Child Neurology Foundation's Shields Fellowship Award, the University of Michigan's Janette Ferrantino
Investigator Award, and the Michigan Institute for Clinical \& Health Research (CTSA: UL1RR024986). Somanetics Corp. (Troy, MI) donated the NIRS equipment for research conducted in our neonatal intensive care unit, but had no input into this study's conception, design, data analysis, writing of the manuscript, or the decision to submit the manuscript.

\section{DISCLOSURE}

R. Shellhaas receives research funding from the NIH, the Child Neurology Foundation, and intramural grants from the University of Michigan's Department of Pediatrics and Communicable Diseases. B. Thelen, J. Bapuraj, J. Burns, A. Swenson, M. Christensen, and S. Wiggins report no disclosures. J. Barks receives research funding from the NIH. Go to Neurology.org for full disclosures.

Received December 7, 2012. Accepted in final form March 29, 2013.

\section{REFERENCES}

1. Azzopardi DV, Strohm B, Edwards AD, et al. Moderate hypothermia to treat perinatal asphyxial encephalopathy. N Engl J Med 2009;361:1349-1358.

2. Gluckman PD, Wyatt JS, Azzopardi D, et al. Selective head cooling with mild systemic hypothermia after neonatal encephalopathy: multicentre randomised trial. Lancet 2005;365:663-670.

3. Shankaran S, Laptook AR, Ehrenkranz RA, et al. Whole body hypothermia for neonates with hypoxic-ischemic encephalopathy. N Engl J Med 2005;353:1574-1584.

4. Toet MC, Lemmers PMA, van Schelven LJ, van Bel F. Cerebral oxygenation and electrical activity after birth asphyxia: their relation to outcome. Pediatrics 2006;117: 333-339.

5. Owens GE, King K, Gurney JG, Charpie JR. Low renal oximetry correlates with acute kidney injury after infant cardiac surgery. Pediatr Cardiol 2011;32:183-188.

6. Cortez J, Gupta M, Amaram A, et al. Noninvasive evaluation of splanchnic tissue oxygenation using near-infrared spectroscopy in preterm neonates. J Matern Fetal Neonatal Med 2011;24:574-582.

7. Urlesberger B, Grossauer K, Pocivalnik M, et al. Regional oxygen saturation of the brain and peripheral tissue during birth transition of term infants. J Pediatr 2010;157:740-747.

8. al Naqeeb N, Edwards AD, Cowan FM, Azzopardi D. Assessment of neonatal encephalopathy by amplitude-integrated electroencephalography. Pediatrics 1999;103:1263-1271.

9. Hellström-Westas L, Rosen I, Svenningsen NW. Predictive value of early continuous amplitude integrated EEG recordings on outcome after severe birth asphyxia in full term infants. Arch Dis Child Fetal Neonatal Ed 1995;72: F34-F38.

10. Toet MC, Hellström-Westas L, Groenendaal F, Eken P, de Vries LS. Amplitude-integrated EEG 3 and 6 hours after birth in full term neonates with hypoxic-ischaemic encephalopathy. Arch Dis Child Fetal Neonatal Ed 1999;81:19-23.

11. Hallberg B, Grossmann K, Bartocci M, Blennow M. The prognostic value of early aEEG in asphyxiated infants undergoing systemic hypothermia treatment. Acta Paediatr 2010;99:531-536.

12. Thoresen M, Hellström-Westas L, Liu X, de Vries LS. Effect of hypothermia on amplitude-integrated electroencephalogram in infants with asphyxia. Pediatrics 2010; 126:e131-e139.

13. Gunn AJ, Wyatt JS, Whitelaw A, et al. Therapeutic hypothermia changes the prognostic value of clinical evaluation of neonatal encephalopathy. J Pediatr 2008;152:55-58. 
14. Shankaran S, Laptook AR, Tyson JE, et al. Evolution of encephalopathy during whole body hypothermia for neonatal hypoxic-ischemic encephalopathy. J Pediatr 2012; 160:567-572.

15. Ancora G, Maranella E, Grandi S, et al. Early predictors of short term neurodevelopmental outcome in asphyxiated cooled infants: a combined brain amplitude integrated electroencephalography and near infrared spectroscopy study. Brain Dev 2013;35:26-31.

16. Thompson CM, Puterman AS, Linley LL, et al. The value of a scoring system for hypoxic ischaemic encephalopathy in predicting neurodevelopmental outcome. Acta Paediatr 1997;86:757-761.

17. Sarkar S, Donn SM, Bapuraj JR, Bhagat I, Barks JD. Distribution and severity of hypoxic-ischaemic lesions on brain MRI following therapeutic cooling: selective head versus whole body cooling. Arch Dis Child Fetal Neonatal Ed 2012;97:F335-F339.

18. Barkovich AJ, Hajnal BL, Vigneron D, et al. Prediction of neuromotor outcome in perinatal asphyxia: evaluation of MR scoring systems. AJNR Am J Neuroradiol 1998;19: 143-149.

19. Jolliffe IT. Principal Component Analysis, 2nd ed. New York: Springer-Verlag; 2002:10-28.

20. Kaufman SA, Miller SP, Ferriero DM, et al. Encephalopathy as a predictor of magnetic resonance imaging abnormalities in asphyxiated newborns. Pediatr Neurol 2003;28: 342-346.

21. Rhee CJ, Kibler KK, Easley RB, et al. Renovascular reactivity measured by near-infrared spectroscopy. J Appl Physiol 2012;113:307-312.

22. Martin CR, Dammann O, Allred EN, et al. Neurodevelopment of extremely preterm infants who had necrotizing enterocolitis with or without late bacteremia. J Pediatr 2010;157:751-756.

23. Selewski DT, Jordan BK, Askenazi DJ, Dechert RE, Sarkar S. Acute kidney injury in asphyxiated newborns treated with therapeutic hypothermia. J Pediatr 2013; 162:725.e1-729.e1.

24. Sarkar S, Barks JD, Bhagat I, Donn SM. Effects of therapeutic hypothermia on multiorgan dysfunction in asphyxiated newborns: whole-body cooling versus selective head cooling. J Perinatol 2009;29:558-563.

25. Saria S, Rajani AK, Gould J, Koller D, Penn AA. Integration of early physiological responses predicts later illness severity in preterm infants. Sci Transl Med 2010;2: $48 \mathrm{ra} 65$.

26. Goldstein B, Fiser DH, Kelly MM, et al. Decomplexification in critical illness and injury: relationship between heart rate variability, severity of illness, and outcome. Crit Care Med 1998;26:352-357.

27. Hellström-Westas L, Rosen I, de Vries LS, Greisen G. Amplitude-integrated EEG classification and interpretation in preterm and term infants. Neoreviews 2006;7: e76-e87.

28. Shellhaas RA, Gallagher PR, Clancy RR. Assessment of neonatal electroencephalography (EEG) background by conventional and two amplitude-integrated EEG classification systems. J Pediatr 2008;153:369-374.

29. Nash KB, Bonifacio SL, Glass $\mathrm{HC}$, et al. Video-EEG monitoring in newborns with hypoxic-ischemic encephalopathy treated with hypothermia. Neurology 2011;76: 556-562.

30. Wusthoff CJ, Dlugos DJ, Gutierrez-Colina A, et al. Electrographic seizures during therapeutic hypothermia for neonatal hypoxic-ischemic encephalopathy. J Child Neurol 2011;26:724-728.

31. Shah DK, Mackay MT, Lavery S, et al. Accuracy of bedside electroencephalographic monitoring in comparison with simultaneous continuous conventional electroencephalography for seizure detection in term infants. Pediatrics 2008;121:1146-1154.

32. Shellhaas RA, Soaita AI, Clancy RR. The sensitivity of amplitude-integrated EEG for neonatal seizure detection. Pediatrics 2007;120:770-777.

33. Gerrits LC, Battin MR, Bennet L, Gonzalez H, Gunn AJ. Epileptiform activity during rewarming from moderate cerebral hypothermia in the near-term fetal sheep. Pediatr Res 2005;57:342-346.

34. Faraway JJ. Practical regression and ANOVA using R. Available at: http://cranr-projectorg/doc/contrib/FarawayPRApdf. Accessed February 15, 2013.

\section{Save These Dates for AAN CME Opportunities!}

Mark these dates on your calendar for exciting continuing education opportunities, where you can catch up on the latest neurology information.

\section{Regional Conference}

- October 25-27, 2013, Las Vegas, Nevada, Encore at Wynn Hotel

\section{AAN Annual Meeting}

- April 26-May 3, 2014, Philadelphia, Pennsylvania, Pennsylvania Convention Center 